\title{
1.8 DELIRIUM and DEMENTIA
}

\section{DELIRIUM}

Delirium is defined as an acute, transient, global disorder of cognition. In two-thirds of cases, delirium occurs in patients with baseline vulnerability, including those with underlying dementia. ${ }^{1}$ Although up to $30 \%$ of older medical patients experience delirium during hospitalization, this condition is unrecognized in nearly two-thirds of cases. ${ }^{2-4}$ Patients with delirium experience an average increase in length of hospital stay of 8 days and mortality rates that are twice as high as those of patients without delirium. ${ }^{5,6}$ In addition, delirium is associated with high rates of functional and cognitive decline and skilled nursing facility placement after hospitalization. The cost of caring for patients with delirium has a marked impact on individual patients, families, and hospital systems. Hospitalists lead their institutions in the development of screening and prevention protocols for patients at risk for delirium, as well as in the promotion of safe treatment approaches. Hospitalists also develop strategies to operationalize cost-effective delirium prevention programs that improve outcomes.

\section{KNOWLEDGE}

Hospitalists should be able to:

- Define delirium and dementia and distinguish between them.

- Differentiate delirium from other causes of cognitive impairment, confusion, or psychosis.

- Describe the indicated tests required to evaluate delirium.

- Describe the causes of delirium in the hospital setting including environmental and iatrogenic risk factors.

- Identify medications known to precipitate delirium.

- Recognize the indications for specialty consultations.

- Describe methods for the prevention of delirium.

- Explain indications, contraindications, and mechanisms of action of pharmacologic agents used to treat delirium.

- Describe nonpharmacologic therapies to manage delirium.

- Describe the complications of delirium in the hospitalized patient.

- Discuss the multifaceted impact that delirium has on patients and their families.

- Explain goals for hospital discharge, including specific measures of clinical stability for safe care transitions.

\section{SKILLS}

Hospitalists should be able to:

- Predict a patient's risk for the development of delirium on the basis of initial history and physical examination.

- Perform appropriate screening for delirium.

- Develop active strategies to reduce delirium in the hospital setting by identifying known patient risk factors that may precipitate delirium.
- Assess patients with suspected delirium in a timely manner, identify the level of care required, and manage or comanage patients with the primary requesting service.

- Perform a focused evaluation for the underlying etiology of delirium and institute prompt treatment to lessen its severity.

- Determine the best setting within the hospital to initiate, monitor, evaluate, and treat patients with delirium.

- Lead multidisciplinary teams to develop and implement care plans for patients with delirium.

- Develop an appropriate pharmacologic plan to manage delirium.

- Develop an appropriate nonpharmacologic plan to manage delirium.

- Develop an appropriate management plan for patients with delirium in the postoperative setting.

- Document an appropriate treatment plan to reduce mortality, limit the duration of delirium and the time required to control agitation, maintain adequate control of delirium, address complications, and manage cost of treatment.

- Use a patient- and family-centered approach in the care of older inpatients.

- Establish goals and boundaries of care with patients and their families.

- Communicate with patients and families to explain the history and prognosis of delirium.

- Facilitate discharge planning early in the hospitalization, including communicating with the primary care provider and presenting the patient and family with contact information for follow-up care, support, and rehabilitation.

- Communicate with patients and families to explain the goals of care, discharge instructions, and management after hospital discharge to ensure safe follow-up and transitions of care.

\section{ATTITUDES}

Hospitalists should be able to:

- Employ a multidisciplinary approach to the care of patients with delirium that begins at admission and continues through all care transitions.

- Follow evidence-based recommendations to guide diagnosis, monitoring, and treatment of delirium and its causes.

- Value a patient- and family-centered approach in the care of older inpatients.

\section{SYSTEM ORGANIZATION AND IMPROVEMENT}

To improve efficiency and quality within their organizations, hospitalists should:

- Lead, coordinate, and/or participate in multidisciplinary teams to develop early treatment protocols.

- Lead, coordinate, and/or participate in multidisciplinary 
initiatives to implement screening and prevention protocols for patients at risk for delirium.

- Lead, coordinate, and/or participate in multidisciplinary initiatives, which may include collaboration with geriatricians, to promote patient safety and cost-effective diagnostic and management strategies for older patients.

- Engage stakeholders in hospital initiatives to improve safety and quality in the care of patients with delirium. 


\section{DEMENTIA}

Dementia is defined as a chronic, often progressive, decline in cognitive function, eventually limiting daily activities. Dementia is a common comorbidity in the hospitalized older patient. Alzheimer disease is the most prevalent form of dementia in older patients, and it accounts for up to $80 \%$ of cases. ${ }^{7,8}$ More than 5 million persons older than 65 years have Alzheimer disease in the United States. ${ }^{7,8}$ Patients with dementia are at increased risk for delirium, falls, and functional decline during hospitalization. Patients with baseline cognitive impairment have prolonged lengths of stay and complex needs after discharge. Agitation and behavioral symptoms of dementia can be exacerbated in the hospital setting and are often difficult to manage. Care of the patient with dementia requires that hospitalists engage in a multidisciplinary approach to inpatient and transitional care. Hospitalists may also become involved in hospital quality and safety initiatives that pertain to areas such as restraint use and fall prevention.

\section{KNOWLEDGE}

Hospitalists should be able to:

- Define delirium and dementia and distinguish between them.

- Differentiate dementia from other causes of cognitive impairment, confusion, or psychosis.

- Describe the indicated tests required to evaluate dementia.

- Describe the causes of potentially reversible dementias or dementia-like conditions.

- List indications, contraindications, and mechanisms of action of pharmacologic agents used to treat dementia.

- Describe nonpharmacologic therapies to manage dementia symptoms.

- Recognize the indications for specialty consultations.

- Describe the complications of dementia in the hospitalized patient.

- Discuss the multifaceted impact that dementia has on patients and their families.

- Explain goals for hospital discharge including specific measures of clinical stability for safe care transition.

\section{SKILLS}

Hospitalists should be able to:

- Perform appropriate screening for dementia.

- Develop active strategies to reduce development of delirium in patients with dementia in the hospital setting by identifying known patient risk factors that may precipitate delirium.

- Assess patients with suspected dementia in a timely manner, identify the level of care required, and manage or comanage patients with the primary requesting service.

- Assess patients for potentially reversible causes of dementia or dementia-like conditions. Assess severity of cognitive impairment and perform a focused evaluation for the underlying etiology of dementia when appropriate.

- Determine the best setting within the hospital to initiate, monitor, evaluate, and treat patients with dementia.

- Formulate and lead multidisciplinary teams to develop and implement care plans for patients with dementia.

- Develop an appropriate pharmacologic plan to manage dementia.

- Develop an appropriate nonpharmacologic plan to manage dementia.

- Use a patient- and family-centered approach in the care of older inpatients.

- Communicate with patients and families to explain the history and prognosis of dementia.

- Use evidence-based methods and tools to assess patients' medical decision-making capacity.

- Defend patients' right to autonomy when so qualified.

- Facilitate discharge planning early in the hospitalization, including communicating with the primary care provider and presenting the patient and family with contact information for follow-up care, support, and rehabilitation.

- Communicate with patients and families to explain the goals of care, discharge instructions, and management after hospital discharge to ensure safe follow-up and transitions of care.

\section{ATTITUDES}

Hospitalists should be able to:

- Employ a multidisciplinary approach to the care of patients with dementia that begins at admission and continues through all care transitions.

- Follow evidence-based recommendations to guide diagnosis, monitoring, and treatment of dementia and its causes.

- Value a patient- and family-centered approach to educate and engage families and caregivers in the care of older inpatients.

- Responsibly address and respect end-of-life care wishes for patients with advanced dementia.

\section{SYSTEM ORGANIZATION AND IMPROVEMENT}

To improve efficiency and quality within their organizations, hospitalists should:

- Lead, coordinate, and/or participate in multidisciplinary teams to develop early treatment protocols.

- Lead, coordinate, and/or participate in multidisciplinary initiatives to implement screening and prevention protocols for patients at risk for poor outcomes related to dementia.

- Lead, coordinate, and/or participate in multidisciplinary initiatives, which may include collaboration withgeriatricians, to promote patient safety and cost-effective diagnostic and management strategies for older patients.

- Engage stakeholders in hospital initiatives to improve safety and quality in the care of patients with dementia.

\section{References}

1. Cole MG. Delirium in elderly patients. Am J Geriatr Psychiatry. 2004;12(1):7-21.

2. Inouye SK. The dilemma of delirium: clinical and research controversies regarding diagnosis and evaluation of delirium in hospitalized elderly medical patients. Am J Med. 1994;97(3):278-288.

3. Inouye SK, Rushing JT, Foreman MD, Palmer RM, Pompei P. Does delirium con- 
tribute to poor hospital outcomes? A three-site epidemiologic study. J Gen Intern Med. 1998;13(4):234-242.

4. Francis J. Delirium in older patients. J Am Geriatr Soc. 1992;40(8):829-838.

5. Cole MG, Primeau FJ. Prognosis of delirium in elderly hospital patients. CMAJ. 1993;149(1):41.

6. McCusker J, Cole M, Abrahamowicz M, Primeau F, Belzile E. Delirium predicts 12-month mortality. Arch Intern Med. 2002;162(4):457-463.

7. Alzheimer's Association. 2014 Alzheimer's disease facts and figures. Available at: https://www.alz.org/downloads/Facts_Figures_2014.pdf. Accessed July 2015.

8. Hebert LE, Scherr PA, Bienias JL, Bennett DA, Evans DA. Alzheimer disease in the US population: prevalence estimates using the 2000 census. Arch Neurol. 2003;60(8):1119-1122. 